\title{
A 60 GHz Dual-Port Probe for Spherical Near-Field Antenna Measurements
}

\author{
Muntianu, Paula Irina; Breinbjerg, Olav
}

Published in:

IEEE Antennas and Wireless Propagation Letters

Link to article, DOI:

10.1109/LAWP.2020.3004883

Publication date:

2020

Document Version

Peer reviewed version

Link back to DTU Orbit

Citation (APA):

Muntianu, P. I., \& Breinbjerg, O. (2020). A 60 GHz Dual-Port Probe for Spherical Near-Field Antenna Measurements. IEEE Antennas and Wireless Propagation Letters, 19(9), 1467 - 1470.

https://doi.org/10.1109/LAWP.2020.3004883

\section{General rights}

Copyright and moral rights for the publications made accessible in the public portal are retained by the authors and/or other copyright owners and it is a condition of accessing publications that users recognise and abide by the legal requirements associated with these rights.

- Users may download and print one copy of any publication from the public portal for the purpose of private study or research.

- You may not further distribute the material or use it for any profit-making activity or commercial gain

- You may freely distribute the URL identifying the publication in the public portal

If you believe that this document breaches copyright please contact us providing details, and we will remove access to the work immediately and investigate your claim. 


\title{
A $60 \mathrm{GHz}$ First-Order Dual-Port Probe for Spherical Near-Field Antenna Measurements
}

\author{
Paula I. Muntianu and Olav Breinbjerg, Fellow, IEEE
}

\begin{abstract}
We present the design, manufacturing, calibration, and testing of a first-order dual-port conical-horn probe for spherical near-field antenna measurements at $60 \mathrm{GHz}$. The measurement results demonstrate that the probe performance is acceptable, but they also illustrate the challenges for highaccuracy antenna measurements at millimeter-wave frequencies.
\end{abstract}

Index Terms-millimeter-waves, antennas measurements, nearfield, dual-polarized probe

\section{INTRODUCTION}

For spherical near-field antenna measurements, first-order dual-port probes possess several advantages; in particular, they ensure an accurate, efficient, and stable near-field-to-far-field transformation using only one full-sphere scan of the antenna under test (AUT) [1], [2]. For these reasons, the DTU-ESA Spherical Near-Field Antenna Test Facility routinely employs first-order dual-port probes from 3-18 GHz. For the higher $\mathrm{mm}$-wave frequencies, dual-port probes are challenged by reduced performances of orthomode transducers (OMT) and switches. Dual-port probes above $40 \mathrm{GHz}$ based on OMTs with a turnstile junction for higher-order modes cancellation with two receiver channels have recently been reported [3], [4]. In this work, we present a first-order dual-port probe based on a high-directivity conical horn, designed and manufactured inhouse, and commercially available cables, OMT, switch, and coax-to-waveguide adapters. We document its design, manufacturing, calibration (S-parameters, radiation pattern, power spectrum, polarization ratio, and channel balance) as well as its performance when employed as probe for measurement of a standard gain horn (SGH). Preliminary results were published in [5], [6]; this work gives a complete account with new and previously unpublished results and analysis.

\section{DESIGN}

The dual-port probe is shown in Fig. 1; it includes a conical horn, a square-to-circular transition $(3.75 \mathrm{~mm}$ to 3.58 $\mathrm{mm}$ ) from Sage Millimeter, a 50-75 GHz OMT from Sage Millimeter with a square antenna port of $3.75 \mathrm{~mm}$, two coaxto-waveguide adapters from Flann Microwave, two 0-67 GHz cables with $1.85 \mathrm{~mm}$ connectors from Flann Microwave, and a $600 \mathrm{MHz}-67 \mathrm{GHz}$ single pole double throw (SPDT) switch from Ducommun. The switch and OMT are selected to have isolation better than $40 \mathrm{~dB}$ in the whole band in order to

P. I Muntianu and O. Breinbjerg are with the Electromagnetic Systems group, Department of Electrical Engineering, Technical University of Denmark, Kongens Lyngby, Denmark (email: paipo@elektro.dtu.dk, ob@elektro.dtu.dk) support a low cross-polarization of the probe. The conical horn is designed, using WIPL-D [7], to have $27 \mathrm{~dB}$ gain in order to partly compensate for the $83 \mathrm{~dB}$ path loss at $60 \mathrm{GHz}$ for the $6 \mathrm{~m}$ measurement distance at the DTU-ESA Facility.

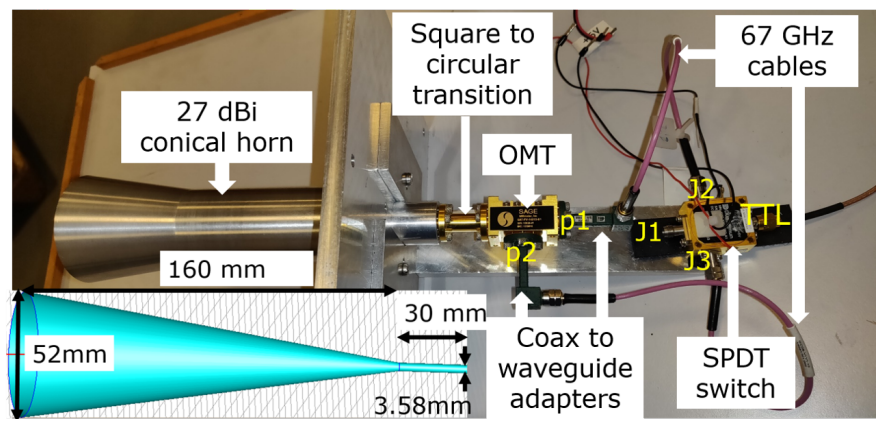

Fig. 1: $60 \mathrm{GHz}$ dual-port probe. For port 1, the switch connects $\mathrm{J} 1$ and $\mathrm{J} 2$, and for port 2, the switch connects $\mathrm{J} 1$ and $\mathrm{J} 3$. The square plate behind the conical horn is part of the standard mounting frame. Horn dimensions are shown in the insert.

\section{Measurement tests}

\section{A. S-parameters}

Fig. 2 shows that the measured S-parameters of the two ports generally have the same frequency variation but also some specific differences; at $60 \mathrm{GHz}$, the $S_{11}$ and $S_{22}$ parameters are $-9 \mathrm{~dB}$ and $-11 \mathrm{~dB}$, respectively. The $S_{21}$ and $S_{12}$ parameters show measured values well below $-40 \mathrm{~dB}$. It has been verified that the S-parameters are largely determined by the switch with little influence of the subsequent components.

\section{B. Radiation pattern}

The dual-port probe radiation pattern is measured without the switch in a spherical near-field facility using a single-port 25 dB SGH (Flann Microwave A6635 series) as probe. The two orthogonal components are measured individually for each probe port, resulting in four full-sphere scans. The measured radiation patterns are shown in Fig. 3 and in Fig. 4, together with WIPL-D simulation results [7]. The radiation patterns are based on the spherical wave expansion with truncation numbers $\mathrm{M}=25$ and $\mathrm{N}=90$ according to the minimum sphere of the dual-port probe.

From the radiation patterns in Fig. 3, and Fig. 4, it is noted that a very good agreement exists between measurements and simulations down to $-20 \mathrm{~dB}$ pattern level for the co-polar 


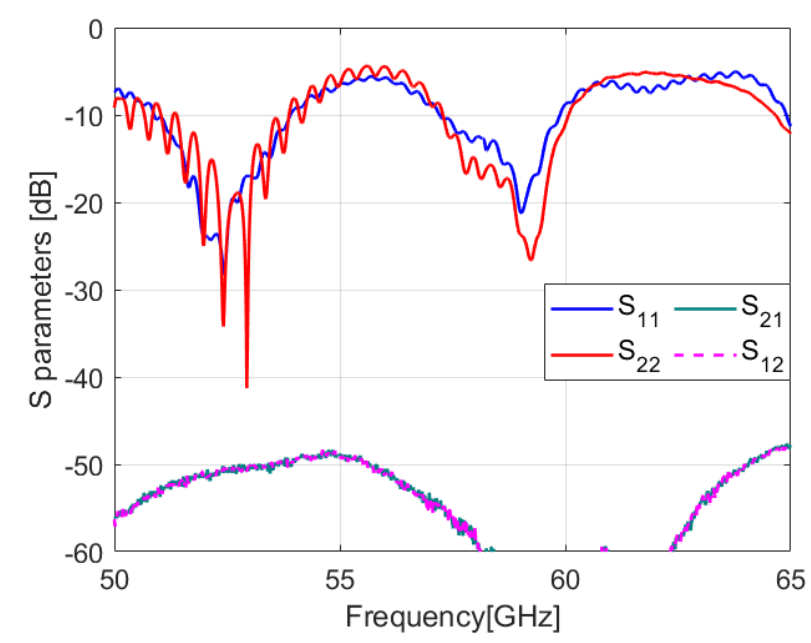

Fig. 2: Measured S-parameters for port 1 and port 2 of dualport probe.
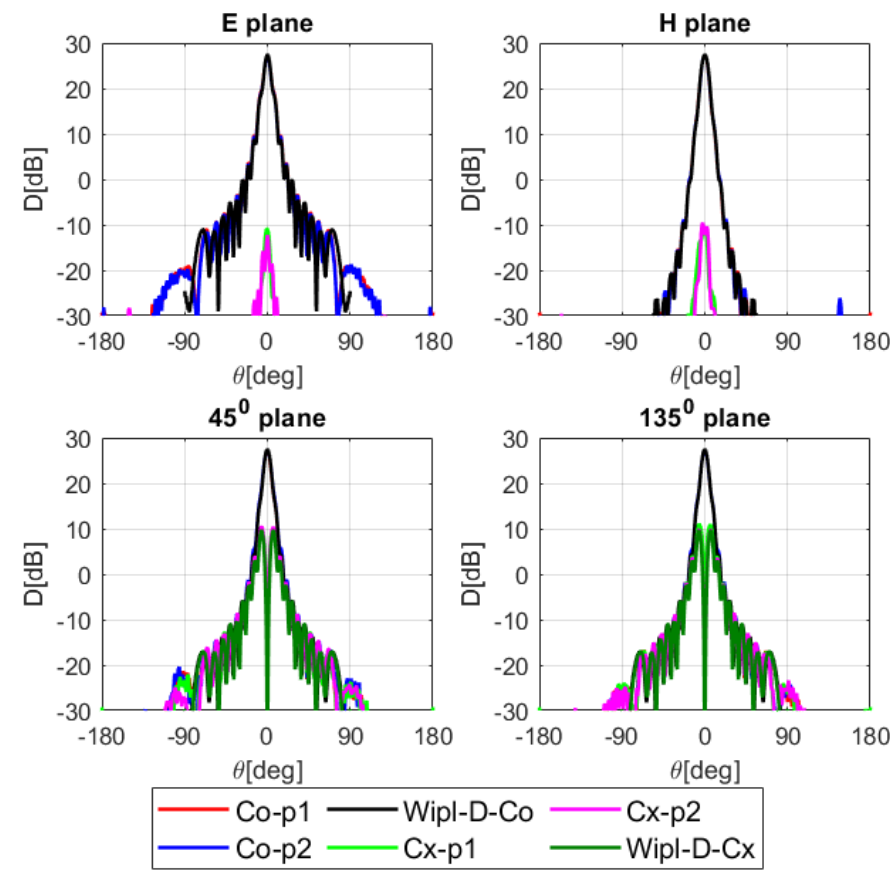

Fig. 3: Measured co-and cross-polar directivity radiation patterns for dual-port probe at $60 \mathrm{GHz}$ for E-plane (top left), $\mathrm{H}$-plane (top right), $45^{\circ}$-plane (bottom left), and $135^{\circ}$-plane (bottom right).

component for the E- and H-planes. The simulations do not exhibit a cross-polar component. The measurement results show an on-axis agreement between the two ports for the cross-polar component with a level of around $38 \mathrm{~dB}$ below the co-polar peak. The measured and simulated data show a very good agreement in the $45^{\circ}$ - and $135^{\circ}$-planes for the copolar and cross-polar components. The reason is that while the cross-polar maximum in the $45^{\circ}$ - and $135^{\circ}$-planes has a slow variation with the azimuthal angle $\phi$, the cross-polar minimum in the E- and H-planes has a fast variation with $\phi$ and is thus more sensitive to accurate definition of coordinate system and
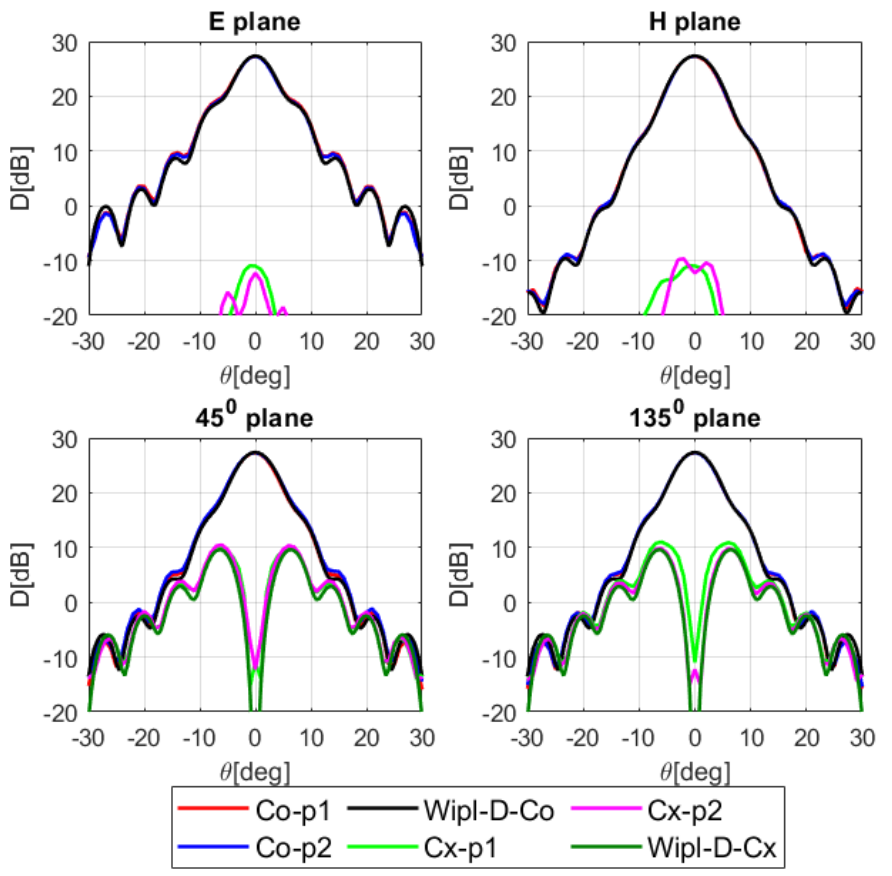

Fig. 4: Zoomed-in measured co-and cross-polar directivity radiation patterns for dual-port probe at $60 \mathrm{GHz}$ for E-plane (top left), H-plane (top right), $45^{\circ}$-plane (bottom left), and $135^{0}$-plane (bottom right).

accurate positioning of the AUT positioner during the fullsphere scan.

\section{Probe Spherical Wave Expansion}

To verify the first-order performance, the measured power spectrum of the spherical $m$ - and $n$-modes is shown in Fig 5 . It is seen that the power level of the higher-order $m$-modes is lower than $-30 \mathrm{~dB}$ for port 1 and port 2 with a slightly increase in level for the odd-order $m$-modes. A likely cause of the higher-order modes presence in the $m$-modes spectrum at port 2, is a displacement or depointing of the dual-port probe with respect to the coordinate system origin, of the order of $1 \mathrm{~mm}$ or less. The ripples for the higher-order $n$-modes is due to the chosen coordinate system with the origin about 62 $\mathrm{mm}$ inside the aperture of the horn; for an origin close to the aperture, the $n$-mode spectrum would be more narrow and without such ripples (while the $m$-mode spectrum would be unchanged).

Since far most of the power is contained within the $m=1$ mode, demonstrating that this is a good first-order probe. The simulated power spectrum (WIPL-D) is compared with the measured power spectrum. For measurements and simulations the sampling step in $\theta$ is $1^{0}$ and in $\phi$ is $3^{0}$. The measurement results show a good agreement with the $m$-modes power level of the simulations and some differences between measurements and simulations in the $n$-modes. These are likely due to lack of absorbers in the simulations and to the different outer dual-port probe surfaces in measurements and in simulations, where only the inner dual-port probe structure is modelled. 


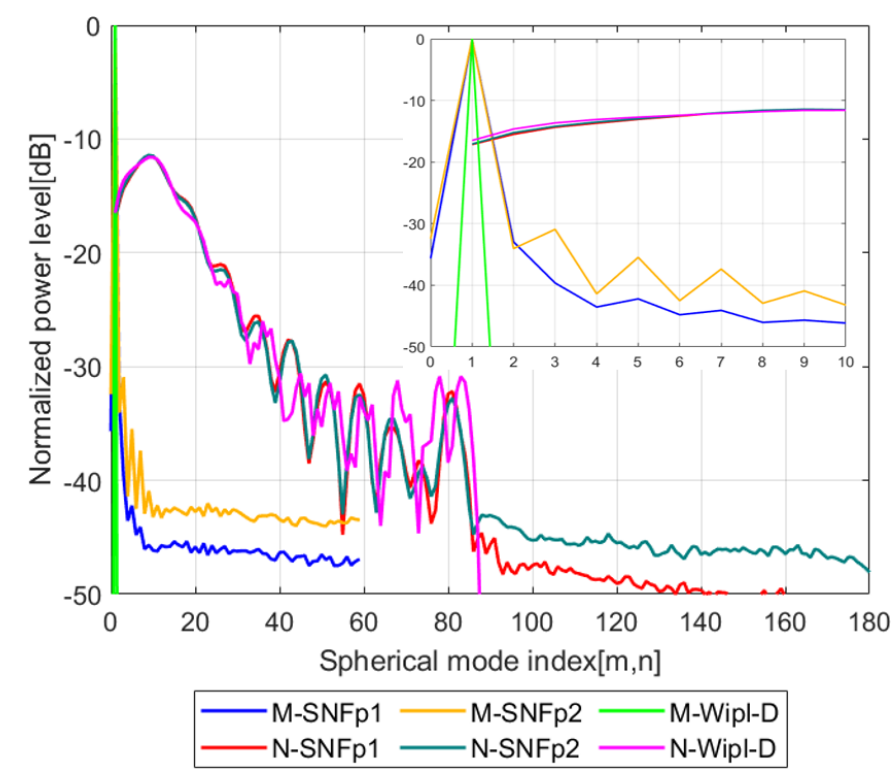

Fig. 5: Dual-port probe $m$ - and $n$-mode power spectra. The zoomed-in plot of the first $10 \mathrm{~m}$ - and $n$-modes is included.

\section{Polarization and channel balance}

The polarization and channel balance calibration is performed using the three antenna measurement procedure [1], wherein the first measurement employs two SGHs and the subsequent ones employ the dual-port probe and each of the two SGHs. This gives one polarization ratio for each probe port and two values for the channel balance; see Table I and Table II.

TABLE I: Polarization calibration

\begin{tabular}{|c|c|c|}
\hline Probe port & $\mathbf{p}_{1}$ & $\mathbf{p}_{2}$ \\
\hline Axial Ratio [dB] & 40.9 & 53.2 \\
\hline${\text { Tilt Angle }\left[{ }^{0} \text { ] }\right.}^{0}$ & -0.1 & 89.3 \\
\hline
\end{tabular}

TABLE II: Channel balance calibration

\begin{tabular}{|c|c|c|}
\hline Channel balance & Magnitude [dB] & Phase [ $\left.{ }^{0}\right]$ \\
\hline $\mathbf{A}_{x y_{1}}$ & 0.02 & 127 \\
\hline $\mathbf{A}_{x y_{2}}$ & -0.15 & 124 \\
\hline
\end{tabular}

A possible explanation for the difference in the channel balance values is the different cross-polarization properties of the two SGHs. In addition, the dual-port probe introduces losses reducing the dynamic range, and noise adds further uncertainty during calibration measurements. Channel balance $\mathrm{A}_{x y_{1}}$ is closer to the ideal value of 1 and this is selected for probe correction of the subsequent measurement results.

\section{E. Gain}

The near-field gain substitution technique is used; a SGH is used as gain reference antenna and this is calibrated with the two-antenna technique [8] to have a gain of $26.4 \mathrm{~dB}$. The dual-port probe gain at $60 \mathrm{GHz}$ gives at port 1 a value of 12.5 $\mathrm{dB}$ and a value of $11.8 \mathrm{~dB}$ at port 2 . These gain values are significantly lower than the $27 \mathrm{~dB}$ gain of the horn itself; this is caused by the losses in the dual-port probe components. The difference between these gain values is due to different OMT port insertion loss values - a $0.4 \mathrm{~dB}$ difference (from OMT datasheet specifications) - and due to the insertion loss at port 2 of the cable, connecting the switch to the OMT, being around $1 \mathrm{~dB}$ larger than for the cable at port 1 .

\section{Dual-Port Probe as a Probe}

The dual-port probe is now tested as a probe for a spherical near-field measurement of a SGH (A6634 series from Flann Microwave) as an AUT. The AUT measured data with dualport probe (DPP) is compared with the data from the measurements when a SGH A6635 (A35) is used as a probe. Fig. 6 and Fig. 7 show the AUT radiation pattern measured with A35 and DPP for the E-, H-, $45^{\circ}$ - and $135^{\circ}$-planes. It can be noted that for measurements with $\mathrm{A} 35$ as probe, the dynamic range of the system (determined as the range from co-polar peak to cross-polar average level), is around $60 \mathrm{~dB}$. When DPP is used as a probe, the dynamic range is around $50 \mathrm{~dB}$, being reduced by approximately $10 \mathrm{~dB}$. This effect is seen in the cross-polar component, showing a noise-like behavior when AUT is measured with DPP. However, the cross-polar on-axis level of the two data sets is the same, with a value of around $-20 \mathrm{~dB}$.
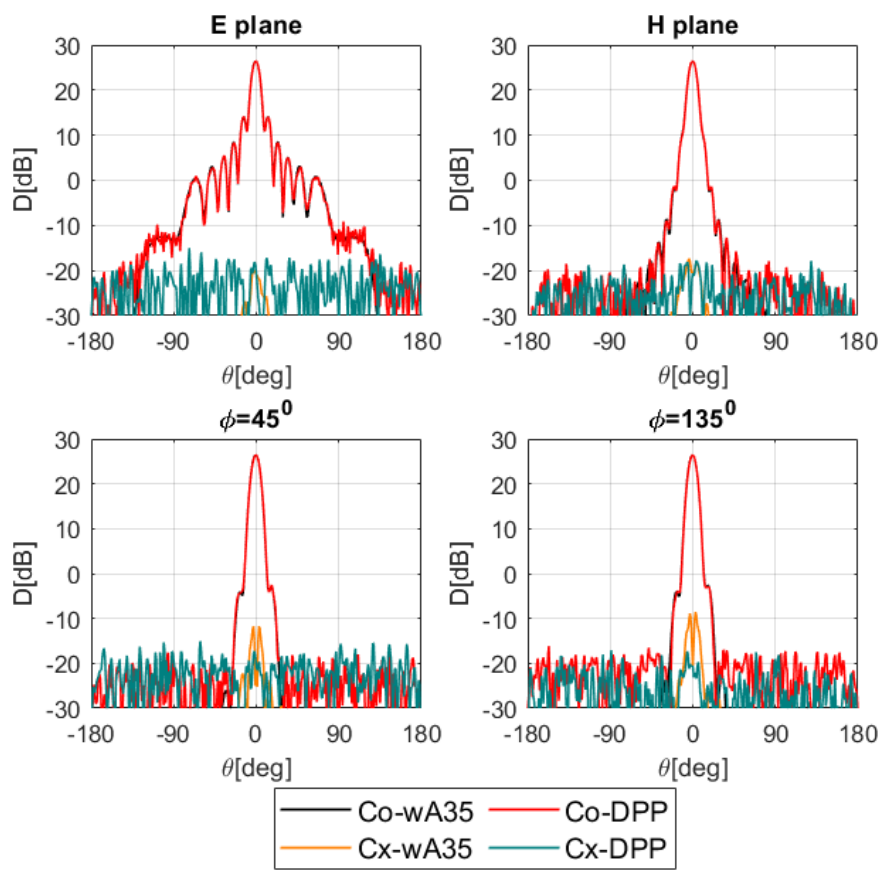

Fig. 6: Measured co-and cross-polar directivity radiation patterns for A6634 SGH at $60 \mathrm{GHz}$ for E-plane (top left), H-plane (top right), $45^{\circ}$-plane (bottom left) and $135^{\circ}$-plane (bottom right).

The results in Fig. 6 and in Fig. 7 show a very good agreement between the measurements obtained with A35 probe and with DPP; the co-polar component showing an agreement down to $-30 \mathrm{~dB}$ pattern levels over the main beam region, while the cross-polar shows the same on-axis level of -20 dB. To quantify the results, the pattern standard uncertainty $\Delta$ 

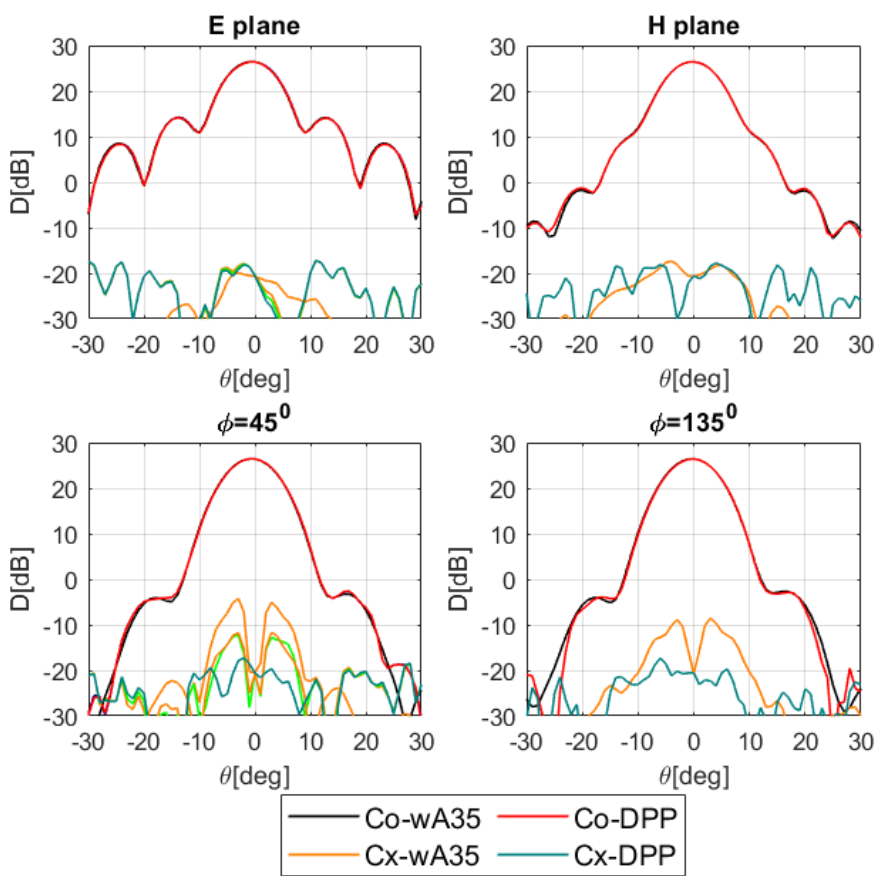

Fig. 7: Zoomed-in measured co-and cross-polar directivity radiation patterns for A6634 SGH at $60 \mathrm{GHz}$ for E-plane (top left), H-plane (top right), $45^{\circ}$-plane (bottom left), and $135^{\circ}$ plane (bottom right).

$(1 \sigma)$, is calculated for the co-polar and cross-polar components for E-, H-planes and for $45^{\circ}$ - and $135^{\circ}$-planes (see Table III). The pattern standard uncertainty is calculated as being $1 / 3$ of the maximum of the equivalent error signal between the two patterns [9]. The standard deviation results for the co-polar component show a very good agreement with hundredths of $\mathrm{dB}$ for E-plane and for $45^{\circ}$ - and $135^{\circ}$-planes and tenths of $\mathrm{dB}$ for H-plane. The cross-polar components show around $2.5 \mathrm{~dB}$ difference between the two measurements results.

TABLE III: Pattern standard uncertainty

\begin{tabular}{|c|c|c|c|c|c|c|c|c|}
\hline \multirow{2}{*}{ Planes } & \multicolumn{2}{|c|}{$\mathbf{E}$} & \multicolumn{2}{|c|}{$\mathbf{H}$} & \multicolumn{2}{|c|}{$\mathbf{4 5}^{0}$} & \multicolumn{2}{|c|}{$\mathbf{1 3 5}^{0}$} \\
\cline { 2 - 9 } & $\mathrm{C}_{o}$ & $\mathrm{C}_{x}$ & $\mathrm{C}_{o}$ & $\mathrm{C}_{x}$ & $\mathrm{C}_{o}$ & $\mathrm{C}_{x}$ & $\mathrm{C}_{o}$ & $\mathrm{C}_{x}$ \\
\hline$\Delta[\mathbf{d B}]$ & 0.05 & 2.2 & 0.1 & 2.5 & 0.03 & 2.3 & 0.03 & 2.5 \\
\hline
\end{tabular}

When the AUT is tested with the dual-port probe, channel balance correction with different values - $\mathrm{A}_{x y_{1}}$ or $\mathrm{A}_{x y_{2}}$ - gives different cross-polarization components for $45^{\circ}$ - and $135^{\circ}$ planes, but the same on-axis polarization level of $-20 \mathrm{~dB}$ (see Fig. 8). If channel balance $A_{x y_{2}}$ is used for the calibration, the overall level of the cross-polar component is higher than in the case when $\mathrm{A}_{x y_{1}}$ is applied. For the E- and H-planes no differences are noticed. This is due to the fact that $45^{\circ}$ - and $135^{\circ}$-planes are more sensitive to the channel balance than E- and H-planes. For completeness, the data corrected with the channel balance value determined as the average between $\mathrm{A}_{x y_{1}}$ and $\mathrm{A}_{x y_{2}}$ is shown as well.

\section{CONCLUSIONS}

A $60 \mathrm{GHz}$ dual-port probe based on switch technology has been designed, manufactured, calibrated, and tested at the
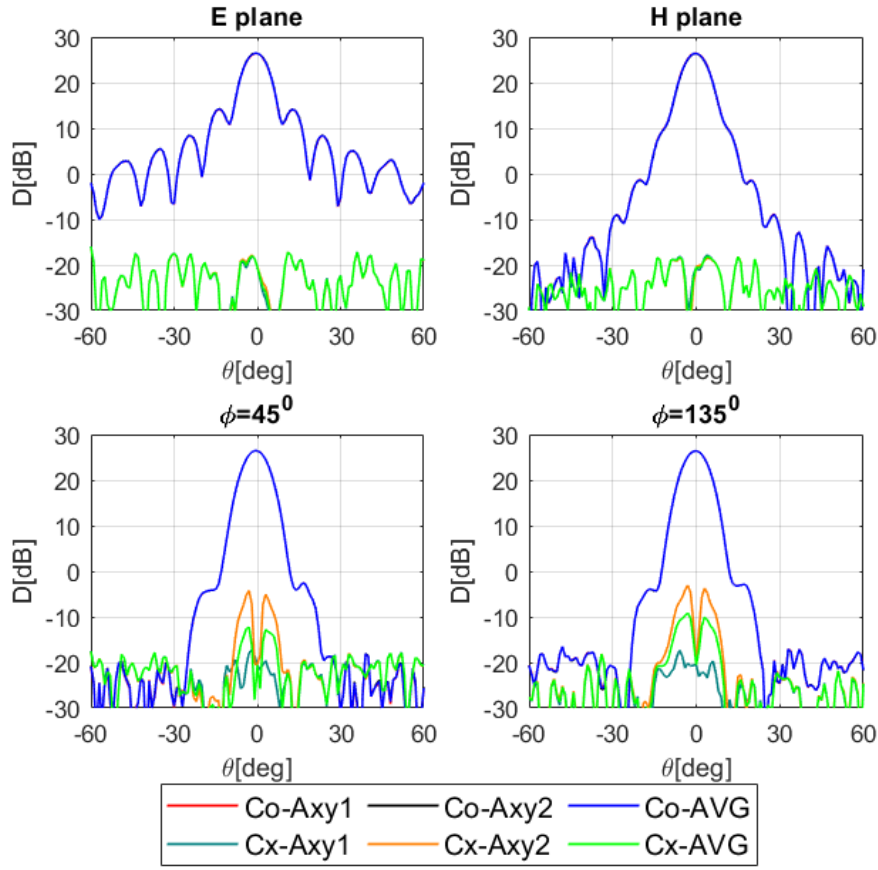

Fig. 8: Measured co-and cross-polar directivity radiation patterns for A6634 SGH at $60 \mathrm{GHz}$ processed with channel balances $\mathrm{A}_{x y_{1}}, \mathrm{~A}_{x y_{2}}$ and with the average value of $\mathrm{A}_{x y_{1}}$ and $\mathrm{A}_{x y_{2}}$ for E-plane (top left) and H-plane (top right) and for $45^{\circ}$-plane (bottom left) and $135^{\circ}$-plane (bottom right).

DTU-ESA Spherical Near-Field Antenna Test Facility. The dual-port probe shows a good first-order performance, with the higher-order $m$-modes power level lower than $-30 \mathrm{~dB}$ at port 1 and port 2. Channel balance calibration is important for mm-waves measurements, but the process at $60 \mathrm{GHz}$ is more challenging and affected by the reduced dynamic range and sources of uncertainties such as mechanical alignment, which here become more significant. The measurement results when dual-port probe is tested as a probe and when a $25 \mathrm{dBi}$ SGH is used as probe show a generally good agreement - within 0.05 $\mathrm{dB}$ (E-plane) and $0.1 \mathrm{~dB}$ (H-plane) for the co-polar component and within $2.5 \mathrm{~dB}$ for the cross-polar component. The dualport probe introduces losses which reduces the dynamic range. This effect is more dominant in the cross-polar component where the larger differences between the two data sets are present. Overall, this work demonstrates that the dual-port probe can be used to conduct accurate measurements at 60 $\mathrm{GHz}$, but it is limited by the switch technology.

\section{REFERENCES}

[1] J. E. Hansen, Spherical Near-Field Antenna Measurements. London, United Kingdom: Peter Peregrinus Ltd, 1988.

[2] 1720-IEEE Recommended Practice for Near-Field Antenna Measurements, APS/SC-Antennas and Propagation Standards Committee, 2012.

[3] L. J. Foged, A. Giacomini, R. Morbidini, V. Schirosi and S. Pivnenko, "Dual Polarized Near Field Probe Based on OMJ in Waveguide Technology Achieving More Than Octave Bandwidth" in 36th Annual Symposium of Antenna Measurements Technique Association, Tucson, AZ, USA, 2014.

[4] A. Giacomini, L. J. Foged, E. Szpindor, W. Zhang, and P. O. Iversen, "High Performance Dual Polarized Near-Field Probe at V-band Provides Increased Performances for Millimeter Wave Spherical Near-Field 
Measurements" in 39th Annual Symposium of Antenna Measurements Technique Association, Atlanta, GA, USA, 2017.

[5] P. I. Popa and O. Breinbjerg, "A $60 \mathrm{GHz}$ dual-polarized probe for spherical near-field measurements" in 39th Annual Symposium of Antenna Measurements Technique Association, Atlanta, GA, USA, 2017.

[6] P. I. Muntianu and O. Breinbjerg, "Comparison of Planar and Spherical Near-Field Antenna Measurements for a $60 \mathrm{GHz}$ Dual-Polarized Probe and $60 \mathrm{GHz}$ Offset Reflector Antenna" in 13th European Conference on Antennas and Propagation, EuCAP, Krakow, Poland, 2019.

[7] WIPL-D homepage, WIPL-D d.o.o. Belgrade, Serbia [Online]. Available: https://wipl-d.com/.

[8] IEEE Standard Test Procedure for Antennas, ANSI/IEEE, Std. 149-1979, The Institute of Electrical and Electronics Engineers Inc., 1979.

[9] A. C. Newel, "Techniques for reducing the effect of measurement errors in near-field antenna measurements" in The Second European Conference on Antennas and Propagation, EuCAP, Edinburgh, UK, 2007. 